\title{
PENSAMIENTO EUROPEO Y LATINOAMERICANO EN DIÁLOGO: CLAVES PARA LA FORMACIÓN SOCIAL Y ÉTICA
}

\section{European and Latin American Thought in Dialogue: Keys for Social and Ethics Training}

\author{
Agustín Ortega Cabrera \\ Universidad Femenina Sagrado Corazón: agustinortegac@unife.edu.pe \\ ID ORCID: 0000-0003-4323-3798
}

\begin{abstract}
RESUMEN
En este artículo desarrollamos nuestra ponencia presentada en el XVII Congreso Nacional de Filosofía, "Reflexión crítica, enseñanza de la Filosofía y crisis de la humanidad" (Huánuco 2019), titulada: "Pensamiento europeo, personalismo y filosofía latinoamericana. Aportaciones para una ética social en un humanismo solidario e integral". Exponemos el dialogo y encuentro entre una serie de corrientes de pensamiento y culturales e inspiradas en la fe como el europeo u occidental, el movimiento obrero, el personalismo o el pensamiento latinoamericano con su trasfondo teológico; con autores como E. Mounier, G. Rovirosa, I. Ellacuría, etc. Lo cual nos proporciona claves y orientaciones para un pensamiento social, una cultura solidaria y ética manifestada en un humanismo integral que posibilita una educación-formación crítica, liberadora y transformadora. En este sentido, se trata de promover valores y principios como la solidaridad, la paz, la justicia, la interculturalidad, la ecología integral junto al buen vivir que orientan a un paradigma o modelo formativo- pedagógico crítico y transformador.
\end{abstract}

\section{Palabras clave:}

Cultura, educación, ética, humanismo, filosofía, pensamiento social, teología.

\begin{abstract}
In this article we develop our presentation presented at the XVII National Congress of Philosophy, "Critical reflection, teaching of Philosophy and crisis of humanity" (Huánuco 2019), entitled: "European thought, personalism and Latin American philosophy. Contributions for a social ethic in a solidary and integral humanism ". We expose the dialogue and encounter between a series of currents of thought and culture inspired by faith such as European or Western, the labor movement, personalism or Latin American thought with its theological background; with authors such as E. Mounier, G. Rovirosa, I. Ellacuría, etc. Which provides us with keys and


guidelines for a social thought, a culture of solidarity and ethics manifested in an integral humanism that enables a critical, liberating and transforming educationtraining. In this sense, it is about promoting values and principles such as solidarity, peace, justice, interculturality, integral ecology together with good living that guide a critical and transformative formative-pedagogical paradigm or model.

\section{Keywords:}

Culture, education, ethics, humanism, philosophy, social thought, theology.

\section{INTRODUCCIÓN. MEMORIA DE LA MILITANCIA OBRERA Y SOCIAL, PASIÓN POR EL IDEAL}

Como ha sido estudiado por la filosofía, por ejemplo, en la teoría crítica con autores como T. Adorno o W. Benjamin, una patología de nuestra época histórica es el olvido o negación de la memoria (Reyes Mate, 2003, 2007). La "memoria passionis" del sufrimiento e injusticia, que se realiza en la experiencia actual de la com-pasión y justicia liberadora con las víctimas de la historia, con los pobres de la tierra y los "testigos" (mártires). Ese compromiso y militancia por un mundo mejor, más libre, justo y fraterno. Una de esas realidades que hay que re-cordar, llevar en el corazón y hacer memoria es la del movimiento obrero y social, con sus militantes ejemplares e inspirados en la fe (Porcar, 1999). Tal como fueron, por ejemplo, Simone Weil, Enmanuel Mounier, Lorenzo Milani, Eugenio Merino, Guillermo Rovirosa, Tomás Malagón, Karol Wojtyła (San Juan Pablo II), I. Ellacuría o Dorothy Day.

Todos estos hombres y mujeres o tantos militantes anónimos que se desconocen y podríamos citar, con sus luces y sombras (aciertos o errores), desde sus diversos ideales o motivaciones éticas, políticas, culturales, espirituales y religiosas: nos presentan una serie de rasgos y caracteres comunes, compartidos que debemos tener en la memoria. Y que son esenciales para el pensamiento, la cultura y la educación, para todo educador e intelectual comprometido, militante en la transformación social, cultural y educativa. Estas claves comunes nos dan el perfil e ideal de un más auténtico compromiso y militancia, tal como transmitió lo más valioso o bueno, verdadero y bello de todo este movimiento obrero y social.

Lo primero que queremos resaltar es que estas personas comprometidas y militantes entregaron toda su vida de forma desinteresada (a fondo perdido), gratuita y radical (profunda) a estos valores e ideales solidarios y de justicia con los obreros, los pueblos y pobres de la tierra. Y pusieron estos valores e ideales por encima de cualquier ideología e ideologización de la realidad, que para ellas no eran absolutas, sino lo principal de dichos valores e ideales para unas relaciones fraternas, justas y solidarias con los otros seres humanos que es lo esencial.

Frente a todo sectarismo e integrismos, era una mentalidad abierta, cooperativa y solidaria que valoraba todo lo bueno, verdadero y bello del otro, que buscaba la unión en los valores e ideales compartidos con los otros. Y que respeta las diversas creencias, ideas y diferencias sin restar para esta militancia común e ideales o valores compartidos. Se pone en primer lugar la vida y dignidad de la persona, el respeto a la integridad física, moral y social del otro. De ahí 
que la militancia se realizaba desde una lucha activa no violenta y pacífica por la justicia, sin dejarse atrapar por el odio, la venganza y la violencia hacia los otros. Experimentaron en sus propias carnes que, como se sabe, la violencia ensucia el alma y las causas más justas como nos muestra la historia. En esta línea, trataron de evitar el prejuicio y sectarismo con el otro, con sus creencias e ideas, que no deja fructificar la relación, el encuentro y convivencia fraterna.

Es una militancia vivida desde la honradez, moral y espiritualidad o mística con los principios, valores e ideales compartidos, universales que nos constituyen como humanos. Ellos comprendieron y experienciaron muy bien que el problema o la cuestión primera no sólo se centra en la estructura o sistema totalitario e injusto como es el capitalismo, los fascismos o el comunismo coectivista (colectivismo). La cuestión de fondo asimismo es el hombre burgués, individualista, posesivo e insolidario que es esclavizado por los ídolos de la riqueza-ser rico, del poder y poseer, de la violencia y del consumidor, del lujo y tener que se pone por encima del ser persona, fraterna y solidaria. Además, al mismo tiempo que buscaron una sociedad distinta y un mundo renovado, vivieron y promovieron una conversión (cambio) personal, una humanidad nueva con una transformación del ser humano en su mentalidad, conciencia, corazón, alma y espíritu.

Tal como ha estudiado la ciencia social (Álvaro E Garrido, 2003), no sólo basta transformar las estructuras sociales y los sistemas económicos o políticos injustos sino el espíritu que anida en el ser humano y que interactúa con esta realidad socioestructural, la mentalidad y conciencia como puede ser la burguesa, capitalista o burocráticaestatalita. Como nos enseña Mounier, la revolución será económica o no será, pero al mismo tiempo debe ser moral o no es (Domínguez, 2002). De ahí que lo más valioso de toda esta militancia del movimiento obrero y social es una renovada antropología, cultura y ética frente a estos ídolos burgueses e individualistas o egolátricos como la riqueza-ser rico y el poder. Una persona con un estilo de vida sobria, austera y de pobreza solidaria en el compartir la vida, los bienes y las luchas liberadoras por la justicia con los pobres, obreros $\mathrm{u}$ oprimidos.

Como nos enseña el pensamiento personalista o latinoamericano (Beorlegui, 2004; De la Torre Rangel, 2005), con filósofos como el ya citado Mounier o el jesuita mártir I. Ellacuría que recoge muy bien todo este espíritu militante, se trata de impulsar la civilización del trabajo y la pobreza frente a la del capital y la riqueza. Es la primacía de la vida de la persona y del trabajador, que siempre tiene la prioridad sobre el capital (beneficio, mercado, producción...). El ser humano con su trabajo, con su dignidad y derechos como es un salario justo, está por encima de la economía, del estado o del partido que debe servir a las necesidades de la humanidad, al bien común, a la justicia y desarrollo humano e integral de los pueblos.

En esta línea, es la existencia desde la pobreza solidaria en la comunión de vida, de bienes y luchas liberadoras con los parias (pobres) de la tierra. En oposición a los ídolos de la riqueza-ser rico, de la propiedad y del tener que como falsos dioses sacrifican la vida y dignidad de las personas. Esta vida del compartir solidario promoviendo, asimismo, de forma sociopolítica el destino universal 
de los bienes, la justa distribución de los recursos, por encima de la propiedad que no es un derecho absoluto e intocable. La propiedad tiene siempre, a la vez, un carácter personal y social con la equidad en el reparto de los bienes y recursos, para que todos tengan acceso a esta propiedad.

Se observa, pues, cómo este movimiento obrero y social e inspirado en la fe se realizó en esta militancia cultural y moral, con la promoción de una ética solidaria internacionalista en la fraternidad universal con los pobres de la tierra que va más allá (está por encima) de naciones, patrias o fronteras; frente a un nacionalismo burgués, excluyente e insolidario. Una vida asociada con los otros, con el asociacionismo solidario e internacionalista que confluyen y une a todo el tejido de organizaciones o movimientos emancipadores por la liberación integral con los pobres u oprimidos, que va cristalizando en redes globales de solidaridad mundial. Dentro de este asociacionismo, el matrimonio y la familia fue una célula vital de fraternidad solidaria, sociabilidad y militancia. Un matrimonio y familia que se constituía en la fidelidad del amor, valores e ideales de la mujer y el hombre con la vida fecunda de los hijos que se expresa en la misión, el servicio, pobreza solidaria (espiritual-evangélica), compromiso social y militancia por la justicia. En contra de la familia burguesa, posesiva, consumista e individualista encerrada en la egolatría.

Asimismo, este asociacionismo y militancia cultural promovió la lectura, formación y educación integral con la creación de ateneos culturales o casas de la cultura y del pueblo, con editoriales, libros y publicaciones como periódicos o revistas. Se fue consciente de la importancia trascendental de la formación de la conciencia, de la promoción cultural y educativa, de la información u opinión pública con una sensibilización y concienciación en todos estos valores, ideales y militancia. La lectura, educación, formación y cultura es esencial para impulsar el conocimiento y comprensión valorativa de la realidad, para una conciencia ética y pensamiento crítico que nos vaya liberando de toda esclavitud, dominación, opresión e injusticia.

Tenemos así todo este legado fecundo del que podemos aprender y nutrirnos para una cultura, compromiso y militancia más real, para este otro mundo posible y necesario que nos vaya llevando a la realización humana, felicidad y un significado más profundo o trascendente de la vida. Como nos recordaba constantemente uno de estos militantes, J. Gómez del Castillo, podemos perderlo todo menos los ideales, con ellos todo está ganado, ellos nos posibilitarán seguir viviendo con sentido y luchando en una existencia realizada (Díaz Salazar, 1990). Tal como nos muestra, asimismo, todo lo anterior este pensamiento social católico (Sols Lucia 2013), la misma doctrina social de la iglesia.

\section{EL PENSAMIENTO FILOSÓFICO Y PERSONALISTA EN DIÁLOGO CON EL LATINOAMERICANO}

En esteapartado, seguimos exponiendo un humanismo ético, social y espiritual (integral) que oriente al pensamiento, a la cultura y educación. Tal como, por ejemplo, nos muestra la corriente de filosofía y pensamiento conocida como personalismo comunitario. Con autores tan significativos como J. Mariatain, E. Mounier, G. Rovirosa, E. Levinas, Karol 
Wojtyła (San Juan Pablo II), X. Zubiri o el mismo Ellacuría (Burgos, 2012; Díaz, 2004; Rodríguez, 2005). Junto a otros autores y testimonios como P. Freire o L. Proaño $(1977,1993)$, muy en sintonía con todo este pensamiento y dichos autores, reseñamos asimismo a L. Milani. Ellos nos muestran toda esta pedagogía, cultura y pensamiento crítico liberador (Corzo, 2007; Dussel, 2002, 2004; Rodríguez E Medina, 2010). El personalismo, con estos maestros y testimonios como Mounier, ha aportado mucho y bueno a la filosofía, la cultura y al compromiso social (Moreno Villa, 1993, 1997).

Ninguna filosofía es perfecta. Y, en este sentido, el pensamiento personalista puede tener sus carencias o límites que habrá que ser precisados, con una actualización y profundización siempre constante en la historia de la cultura. Más, como ha sido estudiado hasta la saciedad por todo tipo de autores o estudios e investigaciones, es innegable la contribución, tan fecunda e importante, que el personalismo ha hecho en todos estos ámbitos del pensamiento. La filosofía personalista, con sus autores como Mounier, supone toda una revolución antropológica, social y ética para el pensamiento, la cultura y el mundo.

Siguiendo a lo más valioso del humanismo filosófico y cristiano-católico, el personalismo pone a la persona como principio, centro y fin de toda la realidad humana, social e histórica. Es decir, toda ética, relación y estructura o sistema, (por ejemplo) en el campo del derecho o de la política y economía, debe estar al servicio de la vida y dignidad sagrada e inviolable del ser humano con sus derechos y deberes. De esta forma, por su propia inspiración espiritual en la fe como muestra Mounier, el personalismo está inspirado en lo más valioso de dicho humanismo cristiano, católico y liberador. Ya que coloca siempre esta vida y dignidad de la persona, con sus necesidades vitales, por encima de cualquier ley o norma e institución, por más sagrada que se considere. En sintonía con el Evangelio de Jesús, "el sábado se hizo para el hombre, y no el hombre para el sábado" (Mc 2, 27-28).

Desde esta ética y espiritualidad del amor que exige la justicia liberadora con los pobres, el personalismo quiere que tengamos vida y que sea una vida fecunda (Jn 10, 10). En la línea de San Ireneo de Lyon y que actualiza San Óscar Romero, la gloria es que el ser humano y el pobre vivan (Martín-Baró, 1997). Y es que como nos muestra la ciencia social y toda esta filosofía (pensamiento), o la misma teología con G. Gutiérrez e I. Ellacuría, los pobres son los que mueren antes de tiempo, a los que se les niega el amor fraterno y la solidaridad (Sols Lucia, 2013). A los pobres se les aplasta su vida, dignidad y derechos. En realidad, eso es lo que promueve todo este humanismo ético, espiritual e integral como es el personalismo: el profundo estupor respecto al valor y a la dignidad de la persona, clave del Evangelio de Jesús como nos enseña igualmente, por ejemplo, San Juan Pablo II programáticamente en Redemptor Hominis (n.10).

Y es que el personalismo nació como una filosofía y pensamiento teoréticopráctico para la acción en el compromiso militante por la defensa de esta vida y dignidad de la persona (Domingo Moratalla, 1985). En oposición a los sistemas e ideologías que niegan al ser humano y sacrifican a los ídolos del capital y del mercado. Tal como impone el liberalismo economicista con el 
capitalismo, ese estatismo partidista del comunismo colectivista, el colectivismo e idolatrías de la nación y raza, los fascismos o nacionalismos sectarios y excluyentes. Frente a todos estos totalitarismos e ideologías burguesas (elitistas), el personalismo nos propone una verdadera revolución en la que las personas, con sus comunidades o pueblos, y los pobres son los sujetos protagonistas de su promoción liberadora e integral. Los seres humanos no son objetos o cosas y, por tanto, no pueden ser sacrificados a estos falsos dioses e idolatrías del capital-mercado y de la riqueza (ser rico), del estado y del poder. Esas idolatrías del poseer y tener que van contra del ser (vida y existencia en dignidad) de la persona.

Como se observa, desde el personalismo se nos muestra una auténtica antropología y ética para la vida social e histórica, donde la persona es fruto del Don (Gracia) del amor y solidaridad del Otro, de Dios mismo y de los otros. Soy amado, luego existo, como afirma este personalismo (Díaz, 2002). Y, como es de bien nacido ser agradecido, esta Gracia de la vida y del amor lleva a la persona a la interrelación, encuentro y responsabilidad con los otros. En el servicio al bien común, con el compromiso y militancia por la justicia liberadora con los pobres de la tierra. Por tanto, desde el personalismo se nos muestra una auténtica naturaleza humana, en sintonía con lo que la tradición filosófica y el pensamiento, asimismo inspirado en la fe, denomina ley natural.

Esto es, el don del Otro, de Dios y de los otros con el amor nos constituye como seres humanos. Somos seres personales, corporales, comunitarios, sociales, políticos y espirituales cuya vida y dignidad es sagrada e inviolable. Personas enraizadas (religadas) en este Don y realidad, desde la comunión solidaria con el Otro y los otros en la justicia con los pobres, con la naturaleza y todo el cosmos. Abiertas a los principios, valores e ideales, a la espiritualidad y trascendencia, a la vida humanizadora, moral, mística y plena.

Nada ni nadie puede ir en contra de toda esta naturaleza humana y personal, oponerse a la vida, dignidad, corporalidad, sociabilidad y trascendencia (espiritualidad) de la persona. Sería ir en contra de lo más sagrado y trascedente. En afinidad con lo que nos transmitía E. Merino, primer consiliario de la HOAC, con claro sabor personalista: lo que no es honrado no puede ser espiritual ni moral; una vida honrada las 24 horas al día, en este Don del amor (Peláez, 2012). La moral y espiritualidad no pueden estar en contra de la vida, felicidad y alegría del ser humano (Domingo Moratalla, 2009). Sino que asumen toda la vida, dignidad y humanidad de la persona para, lejos de negarla, llevarla a su plenitud. Tal como, de forma similar, nos muestra todo lo anterior Tomás de Aquino que, como se ha estudiado, con su humanismo antropológico, ético y trascendente (integral) es uno de los pilares de todo este personalismo filosófico y espiritual.

El personalismo de Mounier o Ellacuría nos traen la revolución de la civilización del trabajo y la pobreza, frente a la del capital y la riqueza (Nicolás E Samour, 2007; Samour, 2003). El capital, el beneficio y la ganancia, no está antes el que trabajo vivo. La vida digna del trabajador y la persona, con sus derechos como es un salario justo, está por encima del capital. Como se observa, es una economía y trabajo que, con la propiedad, se pone al servicio de 
la vida y necesidades de las personas, del destino universal y socialización de los bienes con la propiedad para el desarrollo humano e integral. Y como raíz de esta civilización del trabajo frente a la del capital, la de la pobreza contra la riqueza.

Es la vida de santidad moral, ética, espiritual y militancia en el amor fraterno que se hace pobreza solidaria con la comunión de vida, bienes y luchas por la justicia liberadora con los pobres de la tierra. En oposición a los ídolos de la riqueza-ser rico, del poder y la violencia, frente a todos estos totalitarismos e individualismos burgueses. Y es que el mal del egoísmo con la "codicia (el amor al dinero) es la raíz de todos los males" (1 Tim, 6, 10). Lo más valioso e importante del personalismo. Con estos pensadores y testimonios como Mounier o Rovirosaen ese camino va el mismo L. Proaño-, es que hicieron vida su filosofía, llevaron su pensamiento a la praxis como medio para la paz y justicia liberadora. Ellos lo pusieron en práctica, siendo testimonios honrados y coherentes en una existencia entregada al Otro (Dios) y a los otros, con la pobreza fraterna en solidaridad para la promoción y liberación integral de las personas, los pueblos y los pobres de la tierra.

\section{PENSAMIENTO HUMANISTA, SOCIAL Y CRÍTICO-LIBERADOR PARA OTRA CIVILIZACIÓN}

Por todo ello, como se puede observar y vamos a seguir desarrollando a continuación, existen hilos conductores que se pueden establecer en el diálogo y encuentro entre el pensamiento europeo u occidental, en especial desde el personalismo, con el del Sur empobrecido como es el latinoamericano. Y todo ello inspirado en la fe cristiana-católica con movimientos tan relevantes como los obreros apostólicos, la JOC o la HOAC, las comunidades eclesiales de base y su espiritualidad liberadora que tanto ha marcado a la teología latinoamericana, en su opción por los pobres y la liberación integral. Con estos pensadores, testimonios y mártires como E. Mounier, G. Rovirosa, E. Merino, T. Malagón, L. Milani, J. B. Metz, H. Cámara, Mons. Romero, R. Grande, L. Proaño, S. Ruiz, I. Ellacuría, I. Martín-Baró con sus compañeros jesuitas mártires de la UCA, G. Gutiérrez y un largo etc.

Hay mucha vida y fecundidad en todo este humanismo espiritual e integral, manifestado en estas realidades culturales u obreras, en dichas experiencias del pensamiento personalista, social y latinoamericano inspirado por la fe e iglesia; con todos estos testimonios y mártires ya mencionados. Lo que puede promover una cultura y alternativas éticas con propuestas sociales que, con su base en toda esta espiritualidad liberadora e integral, puede ir contribuyendo a buscar e ir logrando ese otro mundo posible que tanto anhelamos. Tal como, asimismo, ya apuntamos, nos muestra el pensamiento social cristiano-católico y la conocida como Doctrina Social de la iglesia (DSI), con el magisterio de los Papas como Francisco que, como se repite hasta la saciedad, es el tesoro escondido de la iglesia (Scannone, 1987, 2014). Este tesoro de dicho pensamiento social y DSI es ocultado o hasta manipulado por poderes de todo tipo, con ideologizaciones que lo deforman, añadimos nosotros.

Esta cultura y pensamiento nos comunica la pasión por la realidad, la religación y honradez con lo real, con un conocimiento crítico y transformador de toda esta realidad personal, humana, 
social e histórica. Desde el protagonismo, promoción y liberación integral de las personas, los pueblos y los pobres de la tierra. Las personas, los pueblos y los pobres son sujetos de la vida, cogestores de su existencia y desarrollo liberador e integral, de la fe y misión de la iglesia. Las vocaciones, capacidades y posibilidades (con todos los talentos o carismas) de las personas, los pueblos y los pobres: marcan la realidad de la humanidad, la fe e iglesia para la misión, desarrollo y liberación integral.

Y que, como toda auténtica vocación y humanización liberadora, surge del Don (Gracia) de los otros y del Otro, de Dios mismo que con el regalo de su amor liberador nos va reconciliando, perdonando y humanizando integralmente con la llamada a la conversión, a la espiritualidad y mística de encarnación. Verdaderamente, la entraña de lo real es este Don-Gracia del amor que nos va convirtiendo transformadoramente a la fraternidad solidaria y a la justicia con los otros, con los pueblos y los pobres. El corazón de la realidad es esta mística del encuentro en comunión con los otros y el Otro, con Dios, con la humanidad y los pobres, en esta alteridad personalista, comunitaria, solidaria y liberadora. Un amor de comunión con el Dios que se nos revela en Jesucristo, con su iglesia y sus hermanos predilectos, más pequeños, los pobres de la tierra al servicio de la misión, de la fe y la justicia liberadora.

Las personas y la fe están constituidas por este amor fraterno que se hace misericordia y que, en la compasión cordial, lleva al corazón la realidad de las miserias, los sufrimientos e injusticias que padecen los otros, los pueblos y los pobres. Esta espiritualidad, ética e inteligencia cordial realiza la "memoria passionis", la com-pasión y justicia con las víctimas de la historia, con los pueblos crucificados por el mal e injusticia y los pobres de la tierra (Metz, 2007, 2013). Es la memoria que, en el re-cordar permanente y actual, lleva en el corazón las causas liberadoras de los pobres, oprimidos y mártires de la justicia, su cultura, tradiciones, religiosidad popular y espiritualidad.

Una humanidad e iglesia misionera, en salida hacia las periferias y reverso de la historia, humanidad e iglesia pobre con los pobres, en el servicio de la fe y la justicia liberadora del egoísmo con sus ídolos de la riqueza-ser rico, del tener y del poder. Una espiritualidad y moral que se realiza en el seguimiento de Jesús, sirviendo al Reino de Dios y su justicia liberadora con los pobres de la tierra que se opone a todo este anti-reino e imperialismos de la riqueza-ser rico, del poseer y poder. El don de la Gracia liberadora que se acoge, contempla y se hace acción, praxis histórica y liberadora, lucha por la paz y la justicia en el mundo, compromiso sociopolítico por la transformación de la realidad.

Es la vida teologal con las virtudes de la fe, la esperanza y la caridad que tienen un constitutivo carácter social, público y ético-político. El valor espiritual-ético y virtud teologal de la caridad política que busca la civilización del amor, transformar las causas de los males e injusticias con sus estructuras de pecado, sus mecanismos perversos y el desorden establecido. Desde esta espiritualidad del Don del amor solidario y del principio-misericordia en la opción liberadora con los pobres, se realiza la militancia, el compromiso y profetismo por la utopía real de la civilización del trabajo y la pobreza frente a la del capital y la riqueza-ser rico. La dignidad del trabajo y la economía ética que sirva 
a las necesidades de las personas, al desarrollo humanizador de los pueblos y a la liberación integral con los pobres de la tierra que siempre están antes que el capital, el lucro y beneficio (Alvarenga, 2014; Samour, 2012). Una economía y mercado moral que se fundamente en el principio básico del destino universal de los bienes, la justa distribución de los recursos que tiene la prioridad sobre la propiedad que, para ser legítima y ética, debe servir a esta equidad en el destino común de los bienes; cumpliendo así con esta inherente función social de la propiedad.

De esta forma, se van realizando los valores e ideales que orientan a la política, como es el bien común y la justicia que promueven las condiciones sociales e históricas para asegurar los derechos humanos: la educación y cultura; la sanidad y medicamentos; la alimentación y el agua; la vivienda e infraestructuras imprescindibles como las energías ecológicas o el transporte sostenible. Todo lo anterior, como ya indicamos, supone de forma imprescindible la civilización de la pobreza, esto es, la solidaridad en el compartir y comunión de vida, bienes y acción liberadoras por la justicia con los pobres. Unos estilos de vida austera, sobria y del ser solidarios que, en el compromiso por la justicia con los pobres, se oponen a los ídolos de la riqueza-ser rico, del tener, poseer y consumismo. Lo cual posibilita la paz y la ecología integral en esta comunión con Dios, con los pobres y el planeta-creación.

Se promociona así un auténtico desarrollo humano y el buen vivir, con la ética del cuidado y la justicia ecológica personal (o ecología mental), social y ambiental que se hace cargo del clamor de los pobres y de esa casa común que es el planeta tierra (Cabrera Baz, 2017;
Díaz Salazar, 2016; Domingo Moratalla, 2017; Houtart, F. 2007). No habrá paz ni reconciliación sin esta civilización del trabajo, de la pobreza y la ecología integral que promueve la justicia, la solidaridad y el desarrollo global. Es la mundialización de la paz, la solidaridad y justicia global (socioambiental) frente a la globalización neoliberal del capital, de la guerra y la destrucción ecológica.

\section{CONCLUSIONES}

Por tanto, para concluir, resaltamos ese principio crítico y ético-práctico que debe orientar a toda la realidad, humanidad y mundo: promover la vida en todas sus fases (desde el inicio de la vida con la fecundación-concepción hasta el final), formas y dimensiones. La defensa y promoción de este principiovida con la opción por los pobres, a los que se les niega permanentemente esta vida y dignidad sagrada e inviolable de toda persona, nos lleva a oponernos a este sistema e ideología perversa que domina actualmente. Tal como es el neoliberalismo con el capitalismo, hoy ya global.

Como se muestra en la verdad de la realidad histórica, al igual que el comunismo colectivista o colectivismo leninista-stalinista, con su materialismo economicista e individualismo insolidario el capitalismo es intrínsecamente inhumano, inmoral e injusto. Ya que impide la vida y dignidad de los pueblos, de los pobres y del planeta con sus crecientes y obscenas desigualdades e injusticias sociales, globales y ecológicas que destruyen por hambre, pobreza y contaminación ambiental a las personas, a los pobres y al planeta. En este principio-vida y compromiso por la justicia social (global) y ecológica, pilares fundamentales son la mujer, el 
matrimonio y la familia que en el amor con el hombre de forma fecunda se abren a la vida, a los hijos y a la solidaridad. No hay futuro sin el protagonismo e igualdad de las mujeres, de los matrimonios y familias que dan vida, solidaridad y compromiso por la justicia.

Mujeres, matrimonios y familias misioneras, pobres y solidarias en la militancia por la liberación integral con los pobres de la tierra, las mujeres excluidas y familias empobrecidas; frente a la familia burguesa e individualista atrapadas por los ídolos de la riqueza-ser rico, del tener, hedonismo y consumismo. Por tanto, el Dios de la vida revelado en Jesús nos llama a toda esta existencia en el amor fraterno, en la santidad de la pobreza solidaria y justicia con los pobres. Por la que vamos acogiendo realmente este Don de la Gracia liberadora y de la vida humana, plena y eterna que se consumará en las tierras nuevas y en los cielos nuevos.

\section{REFERENCIAS}

Alvarenga, L. (dir.) (2014). Ignacio Ellacuría. Utopía y crítica. Tirant Humanidades.

Álvaro, J. L. E Garrido, A. (2003). Psicología social: Perspectivas Psicológicas y Sociológicas. Mcgraw-Hill.

Beorlegui, C. (2004). Historia del pensamiento latinoamericano: una búsqueda incesante de la identidad. Deusto.

Burgos, J. M. (2012). Introducción al personalismo. Palabra.

Cabrera Bas, A. (2017). Biofilia, restauración urgente para la vida. RUNAE, Monográfico 01, 30-50.
Corzo, J. L. (2007). Educar es otra cosa. Popular.

De la Torre Rangel, J. A. (2005). Iusnaturalismo, personalismo y filosofía de la liberación. Una visión integradora. MAD

Díaz Salazar, R. (1990) ¿Todavía la clase obrera? HOAC.

Díaz Salazar, R. (2016). Educación y cambio ecosocial. PPC.

Díaz, C. (2002), ¿Qué es el personalismo comunitario? IEM.

Díaz, C. (2004). Pedagogía de la ética social. Para una formación en valores. Trillas.

Domingo Moratalla, A. (1985). Un humanismo del siglo XX: el personalismo. Pedagógicas: 1985

Domingo Moratalla, A. (2009). Ética para educadores. PPC.

Domingo Moratalla, A. (2017). Condición humana y ecología integral. Horizontes educativos para una ciudadanía global. PPC.

Domínguez, X. M. (2002). La revolución personalista y comunitaria en Mounier. Instituto E. Mounier.

Dussel, E. (2002). Ética de la liberación en la edad de la globalización y de la exclusión. Trotta.

Dussel, E. (2004). Hacia una filosofía política crítica. Desclee. 
Houtart, F. (2007). El bien común de la humanidad. IAEN.

Martín-Baró, I. (1997). Psicología de la liberación. Trotta.

Moreno Villa, M. (1993). Filosofía de la liberación y personalismo. Universidad de Murcia.

Moreno Villa, M. (1997). Diccionario de pensamiento contemporáneo. San Pablo.

Nicolás, J. A. E Samour, H. (eds.) (2007). Historia, ética y ciencia. El impulso crítico de la filosofía de Zubiri. Comares.

Peláez J. R. (2012). Del "catolicismo social" a la "mística de la HOAC". D. Eugenio Merino Movilla (18811953). Universidad Pontificia de Salamanca.

Metz, J. B. (2007). Memoria passionis. Sal Terrae.

Metz, J. B. (2013). Por una mística de los ojos abiertos, cuando irrumpe la espiritualidad. Herder.

Porcar, F. (1999). Una historia de liberación: una mirada cultural a la historia del movimiento obrero. HOAC.

Proaño, L. (1977). Desclée.
Proaño, L. (1993). Educación liberadora. Corporación editora nacional.

Reyes Mate, M. (2003). Memoria de Auschwitz. Trotta.

Reyes Mate, M. (2007). Medianoche en la historia. Trotta

Rodríguez, E. (2005). Guillermo Rovirosa Albert (1897-1964): espiritualidad y ética del pensamiento social cristiano. $\mathrm{Voz}$ de los sin voz.

Rodríguez, M. I. E Medina, J. J. (2010). Fundamentos pedagógicos del método de encuesta. Davinci.

Samour, H. (2003). Voluntad de liberación. La filosofía de Ignacio de Ellacuría. Comares.

Samour, H. (2012). Crítica y liberación. Ellacuría y la realidad histórica contemporánea. ADG-N.

Scannone, J. C. (1987). Teología de la liberación y Doctrina Social de la Iglesia.

Scannone, J. C. (2014). El Papa Francisco y la teología del pueblo. Razón y fe, 1395, pp. 31-50.

Sols Lucia, J. (2013). Cinco lecciones del pensamiento social cristiano. Trotta,

Fecha de recepción: 15-07-2020

Fecha de aceptación: 10-11-2020 